\title{
Adesão ao tratamento medicamentoso em pacientes com doenças gastrintestinais crônicas acompanhados no ambulatório de um hospital universitario
}

\author{
Nathalie de Lourdes Souza Dewulf ${ }^{1}$, Rosane Aparecida Monteiro ${ }^{2}$, Afonso Dinis Costa Passos ${ }^{2}$, \\ Elisabeth Meloni Vieira ${ }^{2}$, Luiz Ernesto de Almeida Troncon ${ }^{1 *}$ \\ 'Departamento de Clínica Médica, Faculdade de Medicina de Ribeirão Preto, Universidade de São Paulo, \\ ${ }^{2}$ Departamento de Medicina Social, Faculdade de Medicina de Ribeirão Preto, Universidade de São Paulo
}

\footnotetext{
*Correspondência:

N. L. S. Dewulf

Hospital das Clínicas

Faculdade de Medicina de Ribeirão

Preto

Departamento de Clínica Médica

Universidade de São Paulo

Av. Bandeirantes, 3900

14048-900- Ribeirão Preto - SP, Brazil

E-mail: dewulf@usp.br
}

A adesão ao tratamento medicamentoso nas doenças crônicas é um fator de importância clínica e social. Porém, são escassos os estudos deste tema em doenças gastrintestinais crônicas. Assim, investigou-se a adesão ao tratamento medicamentoso prescrito e os possiveis fatores que a influenciam em pacientes em acompanhamento no ambulatório de Gastroenterologia de um hospital universitário ligado ao Sistema Único de Saúde (SUS) brasileiro. Um estudo transversal, com métodos indiretos para avaliar a adesão, incluiu 110 pacientes, dos quais 72,6\% tinham o medicamento fornecido pelo SUS. Os pacientes foram classificados como tendo alto ou baixo grau de adesão, com base em dois diferentes instrumentos. A entrevista identificou 15 (13,7\%) pacientes com baixo grau de adesão ao tratamento. Porém, o teste de Morisky, classificou 64 (58,2\%) com baixo grau de adesão, sendo este comportamento do tipo não intencional em 78,1\% destes casos. Análises univariada e multivariada mostraram que não houve associação estatisticamente significativa entre o grau de adesão e natureza da doença ou fornecimento dos medicamentos, bem como nenhum dos fatores demográficos, sociais, clínicos ou referentes aos medicamentos. Concluiu-se, então, que, entre os pacientes com doenças digestivas crônicas acompanhados em hospital universitário, é freqüente o baixo grau de adesão ao tratamento medicamentoso prescrito.

\section{INTRODUÇÃO}

Nas doenças crônicas cujo tratamento demanda o uso contínuo de medicamentos, para maior eficácia da terapêu- tica, é de estrita importância haver adesão do paciente ao regime medicamentoso prescrito. A adesão pode ser conceituada como o grau de concordância entre o comportamento de uma pessoa em relação às orientações do médico ou de 
outro profissional de saúde (Haynes, Taylor, Sackett, 1981; WHO, 2003; Ostenberg, Blaschke, 2005). O baixo grau de adesão pode afetar negativamente a evolução clínica do paciente e a sua qualidade de vida, constituindo-se em problema relevante, que pode trazer conseqüências pessoais, sociais e econômicas (Marinker, Shaw, 2003).

Nos ambulatórios dos hospitais universitários brasileiros são atendidos pacientes com doenças de alta complexidade, de natureza crônica e que, não raramente, demandam tratamentos prolongados. Para o tratamento de algumas dessas doenças, os serviços públicos de assistência à saúde que estão integrados ao Sistema Único de Saúde (SUS) podem fornecer os medicamentos aos pacientes, utilizando-se de programas especiais (medicamentos essenciais ou de alto custo, de liberação excepcional), que se baseiam em protocolos clínicos e diretrizes terapêuticas bem estabelecidos (Brasil, 2001; 2002a,b). Assim, o baixo grau de adesão ao tratamento ou a má utilização destes medicamentos pode, também, afetar a otimização do funcionamento do SUS e da utilização dos seus recursos.

A adesão à terapêutica é um fenômeno sujeito à influência de múltiplos fatores que afetam diretamente o paciente. Estes fatores, que podem determinar o comportamento da pessoa em relação às recomendações referentes ao tratamento de sua doença, estão relacionados às condições demográficas e sociais do paciente, à natureza da doença, às características da terapêutica, ao relacionamento do paciente com os profissionais de saúde, bem como a características outras, intrínsecas ao próprio paciente (Vermeire et al., 2001; WHO, 2003).

Para caracterizar a adesão do paciente ao tratamento prescrito, diferentes métodos, diretos e indiretos, têm sido utilizados. Os métodos diretos caracterizam-se por permitir detectar os medicamentos, ou os produtos da sua metabolização, nos fluidos biológicos do paciente (Haynes, Taylor, Sackett, 1981; Vermeire et al., 2001). Os métodos indiretos incluem processos de medida feitos por meio de entrevistas com o paciente, informações obtidas de profissionais de saúde e familiares dos pacientes, os resultados dos tratamentos ou atividades de prevenção, preenchimento de prescrições e contagem de comprimidos (Haynes, Taylor, Sackett, 1981; Vermeire et al., 2001).

Conquanto grande número de estudos tenha sido desenvolvido com o objetivo de melhor definir os fatores do alto ou baixo grau de adesão ao tratamento medicamentoso, por parte de pacientes portadores de doenças crônicas, como, por exemplo, a hipertensão arterial (Morisky et al., 1982; Cánovas, Saturno, Esteban, 2001; WHO, 2003; Lowry et al., 2005), o diabetes (Krapek et al., 2004; WHO, 2003; Mino-Leon et al., 2005), a tuberculose (Gonçalves et al., 1999; Rennie, Bates; McKelvie, 2001; WHO, 2003), a síndrome da imunodeficiência adquirida (Levy, Field, 2002, WHO, 2003) e a asma brônquica (Brooks et al., 1994; WHO, 2003; Kim et al., 2005) são escassos os estudos com pacientes portadores de doenças digestivas crônicas, sobretudo no Brasil. Assim, o presente estudo teve o objetivo de investigar o grau de adesão ao tratamento medicamentoso prescrito e identificar possíveis causas associadas ao eventual baixo grau de adesão, dos pacientes em acompanhamento no ambulatório de Gastroenterologia de um hospital universitário ligado ao SUS, do Sudeste do Brasil.

\section{PACIENTES E MÉTODOS}

O estudo foi realizado no Ambulatório de Gastroenterologia (Departamento de Clínica Médica) do Hospital das Clínicas da Faculdade de Medicina de Ribeirão Preto da Universidade de São Paulo (HCFMRP-USP), autarquia da Secretaria de Estado da Saúde, ligado ao SUS, localizado em Ribeirão Preto, Estado de São Paulo, Brasil. Realizou-se um estudo transversal, utilizando a entrevista estruturada como principal método indireto e quantitativo para avaliar o grau de adesão ao tratamento medicamentoso prescrito. O projeto do estudo foi analisado pelo Comitê de Ética em Pesquisa da instituição, sendo aprovado sem restrições em 30 de junho de 2003, de acordo com o parecer constante do processo número 4964/ 2003. O paciente era informado sobre o estudo por meio da leitura do termo de consentimento livre e esclarecido. Após a obtenção do consentimento, com a assinatura do termo, era, então, realizada a entrevista e os demais procedimentos do estudo.

Foram convidados a participar do estudo 116 pacientes, que estavam em seguimento no ambulatório. Destes, 6 se recusaram a participar e 110 deram seu consentimento. A escolha dos pacientes foi feita considerando como critério principal de inclusão a prescrição de uso contínuo e regular de medicamentos para o tratamento das correspondentes doenças digestivas crônicas. O preenchimento deste critério era demonstrado pelo registro deste dado no prontuário médico, que era examinado com antecedência. Outros critérios de inclusão foram o paciente ter idade igual ou superior a 18 anos, estar em seguimento clínico com consultas regulares agendadas no período do estudo e estar, no dia da entrevista, pelo menos na segunda consulta. Foram excluídos os pacientes com deficiências que impedissem a comunicação, ou que estivessem em condições clínicas que desaconselhassem sua participação no estudo.

As entrevistas foram realizadas uma única vez, logo antes da consulta médica agendada para aquele dia, em local reservado, sendo colhidos dados referentes aos últimos 
30 dias de tratamento. Todas as entrevistas foram realizadas no período de junho de 2003 a dezembro de 2004, no próprio Ambulatório de Gastroenterologia do HCFMRPUSP, sendo conduzidas pelo mesmo entrevistador.

Para a realização das entrevistas e a coleta dos demais dados, foram utilizados dois instrumentos: um questionário estruturado e padronizado, contendo teste específico para o grau de adesão, e um formulário de análise de prontuário, onde eram registrados os dados obtidos quando da análise do prontuário dos pacientes já entrevistados. Estes instrumentos foram desenvolvidos pelos autores, tendo como base estudos já publicados (Haynes, Taylor, Sackett, 1981; Pepe, Castro, 2000; Dunbar-Jacob, Mortimer-Stephens, 2001; Vermeire et al., 2001). Com a aplicação desses instrumentos, obtiveram-se as variáveis demográficas, sociais e clínicas do paciente, bem como os dados sobre as principais características da prescrição do tratamento medicamentoso.

Para verificação da inteligibilidade e clareza do questionário, coerência das perguntas, ordem de seqüência, compreensão do paciente ao ouvir e responder, duração provável da entrevista, bem como para avaliar a capacidade de obtenção de dados consistentes do prontuário do paciente, foram realizados testes preliminares com 18 pacientes $(16,4 \%$ da casuística). Os resultados destes testes permitiram aperfeiçoar o questionário da entrevista e a ficha de avaliação do prontuário e compor as versões definitivas dos instrumentos, que foram, então, empregadas no estudo.

Para identificar o grau de adesão ao tratamento medicamentoso, foram estudados a análise dos medicamentos utilizados e o comportamento admitido em relação ao uso de medicamentos, definido pelo teste de Morisky (Morisky et al., 1982; Morisky, Green, Lavine, 1986). Por meio da análise dos medicamentos utilizados, foi possível verificar se os pacientes estavam tomando adequadamente o medicamento conforme a prescrição médica. Perguntava-se ao paciente: a) se estava fazendo uso de medicamento; b) qual medicamento tomava; c) qual a dose diária do medicamento em uso e d) qual a posologia empregada.
Estas informações eram, então, cotejadas com os dados registrados do prontuário referentes à consulta médica imediatamente anterior à data da entrevista e que continham a prescrição dos medicamentos. A partir dos dados obtidos neste cotejo, os pacientes foram classificados em grupos de baixo grau de adesão e alto grau de adesão. Ressalve-se que, no presente estudo, empregou-se o termo "posologia" com o sentido particular de denotar o número de vezes e horário em que deveriam ser tomados os medicamentos.

Foram classificados como apresentando alto grau de adesão os pacientes que afirmavam tomar os medicamentos prescritos e nome do medicamento corretamente. Neste grupo, foram incluídos os casos em que os dados presentes nas informações prestadas pelos pacientes ou obtidos pelo exame dos prontuários foram insuficientes para a análise de algumas informações quanto à dose ou à posologia. Foram classificados como tendo baixo grau de adesão os pacientes que relataram: a) tomar medicamentos a mais do que os prescritos; b) tomar medicamentos a menos que os prescritos; c) tomar outros medicamentos no lugar dos prescritos e d) não tomar os medicamentos prescritos para o tratamento. Ressalte-se que esta análise foi realizada tendo como referência o tratamento medicamentoso específico para a doença digestiva crônica correspondente ao diagnóstico principal.

O teste de Morisky (Morisky et al., 1982; Morisky, Green, Lavine, 1986) é composto por quatro perguntas, que objetivam avaliar o comportamento do paciente em relação ao uso habitual do medicamento (Quadro I). O paciente é classificado no grupo de alto grau de adesão, quando as respostas a todas as perguntas são negativas. Porém, quando pelo menos uma das respostas é afirmativa, o paciente é classificado no grupo de baixo grau de adesão. Esta avaliação permite, também, discriminar se o comportamento de baixo grau de adesão é do tipo intencional ou não intencional, sendo, também, possível caracterizar pacientes portadores de ambos os tipos de comportamento de baixa adesão (Sewitch et al., 2003).

Os dados colhidos na entrevista e no exame do prontuário foram armazenados em banco de dados específico,

QUADRO I - Perguntas que compõe o teste de Morisky e classificação dos tipos de comportamento de baixo grau de adesão, indicados por respostas afirmativas

\begin{tabular}{|c|c|c|}
\hline$\overline{\text { Perguntas referentes ao teste de Morisky }}$ & Não intencional & Intencional \\
\hline "Você, alguma vez, esquece de tomar o seu remédio?" & $\mathbf{X}$ & \\
\hline "Você, às vezes, é descuidado quanto ao horário de tomar o seu remédio?" & $\mathbf{X}$ & \\
\hline "Quando você se sente bem, algumas vez, você deixa de tomar seu remédio?" & & $\mathbf{X}$ \\
\hline "Quando você se sente mal, com o remédio, às vezes, deixa de tomá-lo?" & & $\mathbf{X}$ \\
\hline
\end{tabular}

(Sewitch, 2003) 
criado no ambiente do programa estatístico Epi-info, versão 3.2, de domínio público (disponível em http:// www.cdc.gov/epiinfo). Como forma de minimizar erros, a entrada dos dados no programa foi realizada com dupla digitação.

Possíveis associações entre baixa adesão ao tratamento medicamentoso e variáveis demográficas, escolaridade, procedência, ocupação, modo de aquisição dos medicamentos e número de medicamentos foram inicialmente verificadas mediante o teste exato de Fisher. Para isso, na análise das diversas variáveis, os sujeitos foram classificados em duas categorias, da seguinte maneira: escolaridade (até ensino fundamental completo versus níveis superiores); estado marital (com parceiro versus sem parceiro); procedência (residentes em Ribeirão Preto versus residentes em outros municípios); ocupação (com renda própria versus dependentes); aquisição da medicação (fornecimento integral pelo SUS versus aquisição pelo paciente); número de medicamentos em uso (apenas um versus quantidades maiores). As variáveis que apresentaram um valor de $\mathrm{p} \leq 0,20$ foram incluídas num modelo de regressão logística não-condicional, testando-se previamente para a existência de interações. Em todas as situações, o limite de significância estatística foi fixado em $\mathrm{p}<0,05$. Nas várias análises, utilizaram-se os programas Epi-info e Stata ${ }^{T M}$.

\section{RESULTADOS}

\section{Características dos Pacientes}

As características demográficas, sociais, clínicas e do tratamento medicamentoso dos pacientes incluídos no estudo são apresentadas na Tabela I. Identificou-se predomínio de homens, com idade cima de 40 anos, casados e com ensino fundamental incompleto. Observou-se que $54,6 \%$ possuíam fonte de renda própria, sendo que $26,4 \%$ tinham vínculo empregatício e $28,2 \%$ eram trabalhadores autônomos. Quanto aos rendimentos, 56,4\% recebiam renda per capita de até um salário mínimo ( $\mathrm{R} \$ 240,00)$. Em $74,1 \%$ dos casos, os medicamentos eram fornecidos integralmente pelo SUS. Quanto ao diagnóstico principal pelo qual o paciente era acompanhado no ambulatório de Gastroenterologia, 50,8\% eram portadores de doenças inflamatórias intestinais (doença de Crohn e retocolite ulcerativa), $21,8 \%$ portadores de pancreatite crônica e $27,4 \%$ eram portadores de afecções digestivas variadas, grupo em que houve predomínio da doença do refluxo gastro-esofágico (40\%). Ainda com relação às características clínicas, $69,1 \%$ dos pacientes entrevistados eram portadores de mais de uma doença. Quanto à doença em tratamento no ambulatório de Gastroenterologia, 50\% dos pacientes estavam em tratamento há mais de cinco anos, a

TABELA I - Características demográficas e sociais dos pacientes incluídos no estudo

\begin{tabular}{lclc}
\hline CARACTERÍSTICAS & $\mathrm{N}(\%)$ & CARACTERÍSTICAS & $\mathrm{N}(\%)$ \\
\hline Idade & $70(63,6)$ & Sexo & Homens \\
Acima de 40 anos & & Ocupação & $70(63,6)$ \\
Estado marital & $70(63,6)$ & Autônomo & $31(28,2)$ \\
Casado & $23(20,9)$ & Com vinculo empregatício & $29(26,4)$ \\
Solteiro & $9(8,2)$ & Do lar & $18(16,4)$ \\
Separado & $6(5,5)$ & Aposentado & $14(12,7)$ \\
Viúvo & $2(1,8)$ & Desempregado & $9(8,2)$ \\
União consensual & & Afastado & $7(6,4)$ \\
Escolaridade & $7(6,3)$ & Estudante & $2(1,8)$ \\
Analfabeto/sabe ler e escrever & $63(57,3)$ & Renda per capita & \\
Fundamental incompleto & $12(10,9)$ & Até um salário mínimo & $62(56,4)$ \\
Fundamental completo & $5(4,5)$ & Acima de um salário mínimo & $42(38,2)$ \\
Ensino médio incompleto & $19(17,3)$ & Sem relato & $6(5,4)$ \\
Ensino médio completo & $4(3,6)$ & Aquisição dos medicamentos & $82(74,4)$ \\
Superior completo & & Fornecido integralmente & $23(20,9)$ \\
Procedência & $28(25,5)$ & Arca com gasto total & $4(3,6)$ \\
Ribeirão Preto & $67(60,9)$ & Gasto parcial & $1(0,9)$ \\
Procedente de outra cidade do Estado de SP & $15(13,6)$ & Ignorado & Não procedentes do Estado de SP \\
\hline
\end{tabular}

Os números entre parênteses correspondem às porcentagens $(\mathrm{N}=110)$. $\mathrm{N}=$ número absoluto de pacientes. $\mathrm{SP}=\mathrm{São}$ Paulo 
maioria $(72,7 \%)$ não apresentava operação prévia e a metade $(50,0 \%)$ tinha sofrido pelo menos uma internação anterior.

A Tabela II mostra que, no que se refere às características do tratamento medicamentoso prescrito especificamente para a doença digestiva crônica correspondente ao diagnóstico principal, houve predomínio do uso de apenas um medicamento. Os medicamentos mais utilizados incluíram aminossalicilatos (sulfassalazina e mesalazina) para o tratamento das doenças inflamatórias intestinais, enzimas pancreáticas e insulina para o tratamento dos pacientes portadores de pancreatite crônica e omeprazol para os pacientes portadores de afecções digestivas variadas.

TABELA II - Número de medicamentos em uso por parte dos 110 pacientes incluídos no estudo

\begin{tabular}{lc}
\hline NÚMERO DE MEDICAMENTO EM USO & $\mathrm{N}(\%)$ \\
\hline Um & $65(59,1)$ \\
Dois & $38(34,5)$ \\
Três & $6(5,5)$ \\
Quatro & $1(0,9)$ \\
\hline
\end{tabular}

Os números entre parênteses correspondem às porcentagens. $\mathrm{N}=$ número absoluto de pacientes.

\section{Adesão ao tratamento medicamentoso}

As proporções de pacientes classificados como apresentando baixo ou alto grau de adesão ao tratamento medicamentoso prescrito são apresentadas na Tabela III. A análise dos medicamentos utilizados pelos pacientes revelou que $15(13,7 \%)$ deles foram classificados em baixa ade- são. Dentre estes 15 pacientes em que foi identificada a abaixa adesão, houve os casos em que os pacientes: tomavam medicamentos a mais do que os prescritos $(13,3 \%)$; tomavam medicamentos a menos que os prescritos $(40 \%)$; tomavam outros medicamentos no lugar dos prescritos $(40 \%)$ e não tomavam os medicamentos prescritos para o tratamento $(6,7 \%)$.

Porém, este perfil foi alterado drasticamente quando avaliado o comportamento do uso habitual do(s) medicamento(s) pelo teste de Morisky. De fato, observouse porcentagem muito maior de pacientes classificados como baixo grau de adesão: 58,2\% (64 pacientes). Entre estes pacientes, $50(78,1 \%)$ apresentavam comportamento de baixa adesão do tipo não intencional, indicando padrões de esquecimento ou de descuido com o horário para tomar o medicamento. Ainda dentre os 64 pacientes classificados como baixa adesão, 9,4\% apresentaram comportamento do tipo intencional, em que o paciente toma a decisão de parar de usar o medicamento quando se sente bem, ou quando se sente mal com o uso. Destaque-se que em $12,5 \%$ dos casos classificados como baixa adesão, ambos os tipos de comportamento, intencional e não intencional, foram identificados.

\section{Fatores que afetam à adesão}

Não houve relação entre o grau de adesão ao tratamento e a doença digestiva que o paciente era portador. Por exemplo, houve semelhanças entre os portadores de pancreatite crônica e os pacientes com afecções digestivas variadas quanto ao percentual de casos classificados como menos aderentes, tanto na análise dos medicamentos utilizados $(8,4 \%$ versus $16,6 \% ; p=0,44)$, como nos resultados do teste de Morisky (54,2\% versus $66,7 \% ; p=0,40)$.

TABELA III - Dados sobre adesão ao tratamento medicamentoso prescrito dos pacientes incluídos no estudo, referente à análise dos medicamentos utilizados e ao teste de Morisky

\begin{tabular}{lccc}
\hline GRAU DE ADESÃO & Análise dos medicamentos utilizados & Teste de Morisky $^{*}$ & \\
\hline Alto grau de adesão & $95(86,3)$ & $45(40,9)$ & \\
Baixo grau de adesão & $15(13,7)$ & $64(58,2)$ & $50(78,1)$ \\
Baixa adesão não intencional & - & & $6(9,4)$ \\
Baixa adesão intencional & - & & $8(12,5)$ \\
Baixa adesão com ambos & - & & $64(100)$ \\
os tipos de comportamento & - & $1(0,9)$ & \\
Sub-total & - & $110(100)$ & \\
Ignorado & $110(100)$ & & \\
TOTAL & & & \\
\hline
\end{tabular}

"Morisky et al., 1982; Morisky, Green, Lavine, 1986; os números entre parênteses correspondem às porcentagens dos totais de pacientes; $\mathrm{N}=$ número absoluto de pacientes. 
Não houve, também, relação entre o grau de adesão ao tratamento medicamentoso prescrito e o fato de o paciente ter ou não o fornecimento do medicamento garantido pelo SUS. De fato, não houve diferenças estatisticamente significativas entre os que recebiam, como os pacientes portadores de doenças inflamatórias intestinais, e os que não recebiam o medicamento gratuitamente, como os pacientes com afecções digestivas variadas, quanto ao percentual de casos classificados como menos aderentes, tanto na análise dos medicamentos utilizados $(14,3 \%$ versus $16,6 \% ; p=0,76)$, como nos resultados do teste de Morisky $(57,1 \%$ versus $66,7 \% ; p=0,15)$

Do mesmo modo, nas análises univariadas e multivariadas realizadas, nenhuma das variáveis demográficas, sociais, clínicas ou da terapêutica, apresentou associação estatisticamente significativa com o grau de adesão ao tratamento prescrito.

Assim, não foi possível identificar fator determinante do estado de baixa adesão ao tratamento medicamentoso prescrito.

\section{DISCUSSÃO}

Neste estudo sobre a adesão ao tratamento medicamentoso prescrito a pacientes com doenças digestivas crônicas, avaliando-se o uso do medicamento associado ao conhecimento do nome do remédio pelo paciente, verificouse indício de menor adesão em proporção relativamente baixa $(13,7 \%)$ dos pacientes estudados. Avaliando-se, porém, o comportamento dos pacientes, em relação ao uso do medicamento, por meio do teste de Morisky, observou-se que mais da metade dos pacientes apresentou comportamento indicativo de baixo grau de adesão $(58,2 \%)$, com predominância do tipo não intencional (descuido e/ou esquecimento). Estes achados indicam alto grau de disposição para o uso do medicamento, por parte dos pacientes, como também grau satisfatório de conhecimento, em relação ao nome do medicamento em uso. Indicam, porém, baixo grau de preocupação dos pacientes em relação à utilização correta do medicamento.

Neste estudo, foram utilizados métodos indiretos de avaliação do grau de adesão ao tratamento medicamentoso. Utilizou-se a entrevista, que é um instrumento de fácil aplicação, tem boa aceitação pelos participantes, como também apresenta custo reduzido (Haynes, Taylor, Sackett, 1981). Porém, na análise dos dados obtidos com a entrevista, devem-se levar em consideração as limitações intrínsecas a este método. A análise de diversos estudos em que se compararam os índices de adesão obtidos por métodos diretos e indiretos, observou-se que o paciente aderente dificilmente informa não-adesão ao tratamento (Haynes,
Taylor, Sackett, 1981). Do mesmo modo, o paciente que se declara não-aderente, provavelmente, está descrevendo a verdade (Haynes, Taylor, Sackett, 1981). Porém, quando o paciente informa que está fazendo o uso correto da medicação, conforme o prescrito, e informa corretamente o nome do medicamento, nem sempre se pode garantir a adesão. Isto pode ocorrer porque os pacientes indagados sobre o uso de medicamentos apresentam tendência a relatar comportamento de acordo com o esperado (Haynes, Taylor, Sackett, 1981; Ostenberg, Blaschke, 2005). Por isso, a vulnerabilidade dos métodos indiretos associa-se à maior probabilidade de haver a superestimação da adesão e, correspondendo a isso, a subestimação da não-adesão (Haynes, Taylor, Sackett, 1981; Vermeire et al., 2001; Leite, Vasconcelos, 2003; Ostenberg, Blaschke, 2005).

No presente estudo, em que se utilizou a entrevista, as informações sobre o tratamento prestadas pelo paciente foram comparadas com os dados registrados no prontuário e, adicionalmente, procurou-se acrescentar avaliação do conhecimento sobre o medicamento prescrito. Assim, o foco principal deste instrumento foi identificar a não-adesão. Também se objetivou minimizar a superestimação da proporção de pacientes considerados como mais aderentes, um fato recorrente em estudos com a utilização de entrevistas realizadas diretamente com o paciente (Haynes, Taylor, Sackett, 1981; Vermeire et al., 2001; Leite, Vasconcelos, 2003).

O outro instrumento utilizado neste estudo foi o teste de Morisky. Este teste foi elaborado em 1986 (Morisky, Green, Lavine, 1982) e, apesar de ser indireto, possui, diferentemente da entrevista, a característica de superestimar a não-adesão, devido à rigidez dos seus critérios para caracterizar o paciente como mais aderente (Leite, Vasconcelos, 2003). Isso fica mais evidente quando se observa que, em outros estudos, com métodos diversos, a adesão se caracteriza quando há a utilização de $80 \%$ ou mais da prescrição médica (Kane et al., 2003; Shale, Riley, 2003; Ostenberg, Blaschke, 2005).

A literatura sobre o tema indica que a média de nãoadesão ao tratamento medicamentoso, em condições variadas, é de cerca de $50 \%$, variando de 0 a $100 \%$ (Swarstad et al., 1999; Ostenberg, Blaschke, 2005). Estudos realizados com o objetivo de melhor definir os fatores de alto ou baixo grau de adesão ao tratamento, em doenças crônicas como, por exemplo, a hipertensão arterial (Morisky et al., 1982; Canovas, Saturno, Esteban, 2001), tuberculose (Gonçalves et al., 1999; Rennie, Bates, McKelvie, 2001; WHO, 2003), hanseníase (Bakirtzief, 1996) e a asma brônquica (Brooks et al., 1994; Kim et al., 2005), encontraram porcentagem de não-adesão variando de $20 \%$ a $60 \%$ dos casos. Estudos em doenças digestivas ou a elas relaci- 
onadas, como diabetes melito (Paes, Bakker, Soc-Agnic, 1997; Garcia-Perez et al., 2000; Krapek et al., 2004; Mino-Leon et al., 2005), doenças inflamatórias intestinais (Sewitch et al., 2003; Shale, Riley, 2003) e hiperlipidemias (Miller, 1997; Garcia, 2003), encontraram níveis mais baixos de não-adesão do que os verificados no presente estudo, que variaram, em média, $40 \%$. Neste, encontramos que a proporção de baixo grau de adesão, conforme a análise dos medicamentos utilizados, foi de apenas $13,7 \%$, isto é, bem menor que a dos estudos antes relatados, o que sugere ter havido superestimação do grau de adesão, possível conseqüência não só do método empregado, como antes comentado, mas também dos critérios estabelecidos. Assim é que, se o critério para caracterizar o alto grau de adesão fosse a afirmativa do uso, conforme o prescrito, e o conhecimento correto de nome, dose e posologia, a proporção de pacientes menos aderentes seria muito maior.

Por outro lado, em estudos realizados com o teste de Morisky em outros países, utilizando-se o critério em que, se uma das respostas às questões fosse afirmativa, o paciente seria considerado menos aderente, observaram-se valores da proporção de baixa adesão semelhantes aos encontrados no presente estudo. Destaque-se que o próprio estudo original de Morisky, Green e Levine (1986), encontrou taxa de baixo grau de adesão, da ordem de $43 \%$, em amostra de pacientes que faziam uso de medicamentos para hipertensão arterial. Em outro estudo que utilizou o teste de Morisky para avaliar a adesão ao uso de anticoagulantes, encontrou-se baixo grau de adesão em $50 \%$ dos casos (Davis et al., 2005). Em pacientes que faziam uso de medicamentos para diabetes melito do tipo 2, utilizando-se o mesmo teste, encontraram-se níveis de baixo grau de adesão de até 48,8\% (Krapek et al., 2004).

Em nosso país, alguns estudos sobre adesão ao tratamento, utilizando também o teste de Morisky, apresentaram maior variabilidade quanto à freqüência de baixo grau de adesão. Por exemplo, em um estudo com crianças que faziam uso de antibióticos, realizando entrevista com o cuidador da criança, que era, geralmente, a mãe, encontrouse baixo grau de adesão de 25\% (Crozatti, 2002). Em outra investigação, conduzida, também, em Ribeirão Preto, SP, foi encontrada porcentagem de $55,6 \%$ de baixa adesão, em pacientes hiperlipêmicos que faziam acompanhamento em Unidades Básicas de Saúde (Garcia, 2003). Outro estudo realizado na mesma cidade, com pacientes portadores de hipertensão arterial, utilizando o teste de Morisky, classificou como baixo grau de adesão o paciente que respondesse afirmativamente a, no mínimo, duas das questões. Mesmo com este critério menos rígido, o baixo grau de adesão encontrado foi de 54,6\% dos pacientes estudados (Strelec, 2000). Assim, comparando os dados sobre o com- portamento dos pacientes do presente estudo com os achados da literatura, utilizando o teste de Morisky, pode-se perceber que a proporção de casos com baixo grau de adesão ao tratamento foi semelhante aos relatados em outros estudos.

Em relação ao tipo baixa adesão do paciente, observou-se, no presente estudo, por meio do teste de Morisky, que o comportamento do tipo não intencional foi predominante, ou seja, o paciente freqüentemente esquece de tomar o medicamento, ou é descuidado com o horário para tomar os remédios. Destaque-se que o esquecimento é um dos tipos de comportamentos que vem sendo observado em outros estudos, como fator mais freqüente que leva ao baixo grau de adesão ao tratamento medicamentoso prescrito (Dunbar-Jacob, Mortimer-Stenphens, 2001; Kane et al., 2003; Sewitch et al., 2003).

$\mathrm{Na}$ análise dos possíveis fatores relacionados com a adesão, observou-se, em todo o estudo, que não foi encontrado um único fator que tivesse associação estatisticamente significativa com o alto ou baixo grau de adesão ao tratamento. Destaque-se que nem a natureza da doença (diagnóstico), nem o fato de haver fornecimento gratuito dos medicamentos parecem influenciar a adesão ao tratamento. Isso sugere a possível existência de padrão de comportamento comum aos pacientes entrevistados, usuários do Ambulatório de Gastrenterologia do HCFMRP-USP, independente da doença, do fornecimento do medicamento e de outras variáveis de natureza demográfica, socioeconômica ou clínica. Uma possibilidade de estratégia para avaliar esta hipótese seria a utilização de outras abordagens de estudo, como as que envolvem métodos da pesquisa qualitativa, que permitem aprofundar o conhecimento do tema em um determinado grupo, desvendando suas outras características, de natureza mais subjetiva (Leite, Vasconcelos, 2003).

Desta forma, para avaliar a adesão ao tratamento medicamentoso prescrito, deve-se não apenas considerar os fatores externos ao paciente, mas também a sua compreensão do tratamento e a relação estabelecida com os profissionais de saúde. A importância da comunicação e a necessidade dos profissionais de saúde bem conversarem com o paciente, visando transformar as informações por ele recebidas em conhecimentos definidos, uma vez que a falta de conhecimento ou a existência de dúvidas que o paciente guarda para si constituem, também, fatores de não-adesão (Cánovas, Saturno, Esteban, 2001; Levy, Feld, 1999; Pepe, Castro, 2000). Para isto, ações visando estimular a comunicação, que podem ser realizadas em equipes multidisciplinares, devem ser incentivadas, para que haja otimização do trabalho de informação do paciente (Balkrishanan, 2005). Isso possibilitaria que o paciente 
estivesse mais preparado para assumir um papel ativo e responsável em seu tratamento, permitindo, também, que o mesmo percebesse que pode controlar os sintomas com atitudes de maior adesão (Pepe, Castro, 2000; Robinson, 2004; Svarstad, 1999).

Neste estudo, foram analisados possíveis aspectos que afetam a adesão ao tratamento, como os sóciodemográficos e os relacionados à doença e a terapêutica. Porém, destaca-se a necessidade de avaliar, também, fatores relacionados aos profissionais e ao sistema de saúde, bem com os fatores ligados ao próprio paciente. Assim, apesar desses aspectos não terem sido analisados no presente estudo, deve-se considerar o contexto do atendimento, uma vez que é possível identificar casos de falhas na atenção ao paciente. Por se tratar de um hospital escola, a rotatividade na equipe médica que atende os pacientes é alta, o que pode afetar a possibilidade de estabelecimento do vínculo entre o paciente e o médico, fator importante para a existência da confiança por parte do paciente. Esta confiança que o paciente deposita em toda a equipe de saúde, no médico responsável pela prescrição, o farmacêutico que orienta o uso do medicamento, no medicamento prescrito e no tratamento como um todo, é um possível fator de influência sobre o baixo grau de adesão ao tratamento (Leite et al., 2002; Marinker; Shaw, 2003; Pepe, Castro, 2000).

Do mesmo modo, existe, também, grande dificuldade do paciente ter acesso ao farmacêutico, último profissional de saúde a ter contato com o paciente do serviço ambulatorial, antes de iniciar o uso da medicação, para receber orientações sobre o medicamento e o seu uso. Isso pode ser devido ao grande número de pacientes atendidos pela farmácia do hospital, como também por haver a possibilidade da retirada do medicamento por outra pessoa que não o paciente, o que pode caracterizar outra lacuna na comunicação com o paciente.

Desta forma, percebe-se a necessidade de maior atenção voltada para aspectos da atenção à saúde, como a comunicação com o paciente, com maior preocupação no investimento na informação e na educação dos pacientes, por meio de intervenções que permitam que eles se conscientizem sobre a importância do uso correto dos medicamentos. Isto ficou bem evidenciado no presente estudo, uma vez que a utilização do teste de Morisky permitiu demonstrar grau considerável de descuido do paciente quanto à utilização do medicamento prescrito. Igualmente, seria necessário investir na atualização dos profissionais de saúde, discutindo, junto à equipe de saúde, o problema da nãoadesão ao tratamento e das suas causas, bem como as possibilidades da sua resolução. Frente à necessidade de se trabalhar com o paciente de forma abrangente, novamente fica clara a pertinência de um enfoque multidisciplinar, visando à melhoria da qualidade de vida daquele que busca atendimento.

A maior adesão dos pacientes ao tratamento permitiria, também, melhor utilização dos recursos financeiros do Sistema Único de Saúde, pois o uso mais adequado desses medicamentos, que são classificados como "de alto custo", possivelmente reduziria a necessidade de se recorrer a procedimentos diagnósticos e terapêuticos mais custosos.

\section{CONCLUSÕES}

Em pacientes com doenças digestivas crônicas, atendidos em ambulatório especializado de hospital terciário, é freqüente o baixo grau de adesão ao tratamento medicamentoso, havendo predomínio de comportamento não-intencional de baixa adesão (descuido ou esquecimento). O comportamento de baixo grau de adesão parece ser independente de características individuais, da natureza da doença (diagnóstico) ou do fornecimento gratuito do medicamento, podendo tratar-se de traço comum aos usuários do serviço. Isto mostra a necessidade de medidas educacionais visando aumentar a adesão ao tratamento, o que implica em maior atenção dos profissionais da saúde para o uso dos medicamentos pelos pacientes.

\section{ABSTRACT}

\section{Compliance to drug therapy in university hospital outpatients with chronic digestive diseases}

Compliance to drug treatment is an important determinant of patient's clinical evolution and health system expenditure. However, studies on compliance in chronic digestive diseases are scarce. In order to investigate compliance to prescribed medication and factors affecting it, a cross-sectional, indirect study was carried out among 110 outpatients of a University Hospital Gastroenterology clinic. These patients were characterized as presenting either higher or lower degree of compliance by two different instruments. The same classification was made after applying the Morisky test, which is based on patient answers to four standardized direct questions. The interview identified $15(13.7 \%)$ patients as not following correctly the prescribed treatment. However, up to 64 patients $(58.2 \%)$ were classified as less compliant by the Morisky test, which also indicated a non-intentional behavior in 50 (78.1\%) out of these patients. There were no relationships between compliance and either disease nature or free access to medication. Also, univariate and multivariate statistical analysis showed that none of the demographic, social, clinical, or drug-related factors 
presented any statistically significant relationship that could indicate an influence on compliance to treatment. Low compliance to drug treatment is relatively common amongst patients with chronic digestive disease.

UNITERMS: Drug utilization. Patient compliance. Gastroenterology.

\section{REFERÊNCIAS BIBLIOGRÁFICAS}

BAKIRTZIEF, Z. Identificando barreiras para aderência ao tratamento de hanseníase. Cad. Saúde Pública, v. 12, n. 4, p. 497-505, 1996.

BALKRISHANAN, R. The importance of medication adherence in improving chronic-disease related outcomes: what we know and what we need to further know. Med. Care, v. 43, n. 6, p. 517-520, 2005.

BRASIL. Portaria 263. Protocolo clínico e diretrizes terapêuticas para o tratamento de fibrose cística. Diário Oficial da União, 2001; 18 de jul.

BRASIL. Portaria 858. Protocolo clínico e diretrizes terapêuticas para o tratamento de doença de Crohn. Diário Oficial da União, 2002a; 5 de nov.

BRASIL. Portaria 861/02. Protocolo clínico e diretrizes terapêuticas para o tratamento de retocolite ulcerativa. Diário Oficial da União, 2002b; 5 de nov.

BROOKS, CM; RICHARDS, JM; KOHLER, CL; SOONG, S-J; MARTIN, B; WINDSOR, RA; BAILEY, WC. Assessing Adherence to Asthma Medication an Inhaler Regimens: A Psychometric of Adult Self-Report Scales. Med. Care, v. 32, n. 3, p. 298-307, 1994.

CÁNOVAS, J. J. G.; SATURNO P. J.; ESTEBAN, B. L.; Grupo de investigación del proyecto sobre evaluación y mejora de la adhesión terapéutica en la hipertensión. Evaluación y mejora de la adhesión terapéutica en los pacientes hipertensos. Aten. Primaria, v. 28, n. 9, p. 615619, 2001.

CROzATTI, M. T. L. Estudo da utilização de antimicrobianos em infecções respiratórias agudas em crianças atendidas nas unidades de saúde de MaringáPR: adesão e nivel de informação na perspectiva do paciente. São Paulo, 2002. 94 p. [Dissertação de Mestrado. Faculdade de Saúde Pública. Universidade de São Paulo].
DAVIS, N. J.; BILletT, H. H.; COHEN, H. W.; ARNSTEN, J. H. Impact of adherence, knowledge, and quality of live on anticoagulation control. Ann. Pharmacother., v. 39, p. 632-636, 2005.

DUNBAR-JACOB, J; MORTIMER-STEPHENS, MK. Treatment adherence in chronic disease. J. Clin. Epidemiol., v. 54, p. S57-S60, 2001.

GARCIA, RAC. Os fatores de aderência ao tratamento farmacológico de hiperlipidemias em pacientes atendidos pela Secretaria Municipal de Saúde de Ribeirão Preto. Ribeirão Preto, 2003. 104 p. [Dissertação de Mestrado. Faculdade de Medicina de Ribeiro Preto, Universidade de São Paulo].

GARCIA PÉREZ, AM; LEIVA FERNÁNDEZ, F; MARTOS CRESPO, F; GARCIA RUIZ, AJ; PRADOS TORRES, D; SÁNCHEZ DE LA CUESTA Y ALARCÓN, F. Como diagnosticar el cumplimiento terapéutico en atención primaria. Med. Fam., v. 1, n. 1, p. 13-19, 2000.

GONÇALVES, H; COSTA, J.S.D; MENEZES, A.M.B; KNAUTH, D; LEAL, O.F. Adesão à terapêutica da tuberculose em Pelotas, Rio Grande do Sul: na perspectiva do paciente. Cad. Saúde Pública, v. 15, n. 4, p. 777-787, 1999.

HAYNES, R.B; TAYLOR, D.W; SACKETT, D.L. Compliance in health care. Baltimore: Johns Hopkins University Press, 1981. 516 p.

KANE, S.; HUO, D.; AIKENS, J.; HANAUER, S. Medication nonadherence and the outcomes of patients with quiescent ulcerative colitis. Am. J. Med., v. 114, p. 39-43, 2003.

KIM, C.; FELDMAN, H. I.; JOFFE, M.; TENHAVE, T.; BOSTON, R; APTER, A. J. Influences of earlier adherence and symptoms on current symptoms: a marginal structural models analysis. J. Allergy Clin. Immunol., v. 115, n. 4, p. 810-814, 2005.

KRAPEK, K.; KING, K.; WARREN, S. S.; GEORGE, K. G.; CAPUTO, D. A.; MIHELICH, K.; HOLST, E. M.; NICHOL, M. B.; SHI, S. G.; LIVENGOOD, K. B.; WALDEN, S.; LUBOWSKI, T. J. Medication adherence and associated hemoglobin A1c in type 2 diabetes. Ann. Pharmacother, v. 38, p.1357-1362, 2004. 
LEITE, J.C.C.; DRACHLER, M.L.; CENTENO, M.O.; PINHEIRO, C.A.T.; SILVEIRA, V.L. Desenvolvimento de uma Escala de Auto-Eficácia para Adesão ao Tratamento Anti-retroviral. Psicol. Reflex Crit., v. 15, n. 1, p. 121-133, 2002.

LEITE, S.N; VASCONCELOS, M.P.C.Adesão à terapêutica medicamentosa: elementos para a discussão de conceitos e pressupostos adotados na literatura. Ciência \& Saúde Coletiva, v. 8, n. 3, p. 775-782, 2003.

LEVY, R.L; FELD, A.D. Increasing patient adherence to gastronterology treatment and prevention regimens. Am. J. Gastroenterol., v. 94, n. 7, p. 1733-1742, 1999.

LOWRY, K.P.; DUDLEY, T.K.; ODDONE, E.Z.; BOSWORTH, H. B. Intentional and unintentional nonadherence to antihypertensive medication. Ann. Pharmacother., v. 39, n. 7-8, p. 1198-1203, 2005.

MARINKER, M.; SHAW, J. Not to be taken as directed: putting concordance for taking medicines into practice. $B M J$, n. 326, p. 348-349, 2003.

MILLER, N.H. Compliance with treatment regimens in chronic asymptomatic diseases. Am. J. Med., v. 102, p. 4349, 1997.

MINO-LEON, D.; FIGUEIRAS, A.; AMATO, D.; LAPORTE, J.R. Treatment of type 2 diabetes in primary health care: a drug utilization study. Ann. Pharmacother., v. 39, n. 3, p. 441-445, 2005.

MORISKY, D.E.; LEVINE, M.; GREEN, L.W.; SMITH, C.R. Health education program effects on the management of hypertension in the elderly. Arch. Intern. Med., v. 142, n. 10, p. 1835-1838, 1982.

MORISKY, D.E.; GREEN, L.W.; LEVINE, D.M. Concurrent and predective validity of self-reported measure of medication adherence. Med. Care, v. 24, p. $67-74,1986$.

OSTENBERG, L.; BLASCHKE, T. Adherence to medication. N. Engl. J. Med., v. 353, p 487-497, 2005.

PAES, A.; BAKKER, A.; SOE-AGNIE, C.J. Impact of dosage frequency on patient compliance. Diabetes Care, v. 20, p. 1512-1217, 1997.
PEPE, V.L.E.; CASTRO, C.G.S.O. A interação entre prescritores, dispensadores e pacientes: informação compartilhada como possível benefício terapêutico. $\mathrm{Cad}$. Saúde Pública, v. 16, n. 3, p. 815-822, 2000

RENNIE, T.W.; BATES, I.; McKELVIE, W. Compliance and adherence as pragmatic concepts: a study of TB in rural Pakistan. Int. J. Pharm. Pract., v.9, suppl., p. R42, 2001.

ROBINSON,A. Review article: inflammatory bowel disease - empowering the patient and improving outcome. Aliment. Pharmacol. Ther., v. 20, suppl. 4, p. 84-87, 2004.

SEWITCH, M.J.; ABRAHAMOWICZ, M.; BURKUN, A.; BITTON, A.; WILD, G.E.; COHEN, A.; DOBKIN, P.L. Patient nonadherence to medication in inflammatory Bowel disease. Am. J. Gastroenterol., v. 98, n. 7, p. 15351544, 2003.

SHALE, M. J., RILEY, S. A. Studies of compliance with delayed-release mesalazine therapy in patients with inflammatory bowel disease. Aliment. Pharmacol. Ther., v. 18, p. 191-198, 2003.

STRELEC, M. A. A. M. A influência do conhecimento sobre a doença e atitude frente à tomada dos remédios no controle da hipertensão arterial. São Paulo, 2000. $139 \mathrm{f}$. [Dissertação de Mestrado. Escola de Enfermagem. Universidade de São Paulo].

SVARSTAD, B.L.; CHEWNING, B.A.; SLEATH, B.L.; CLAESSON, C. The brief medication questionnaire: A tool for screening patient adherence and barriers to adherence. Patient Education and Counseling, v. 37, p. 113-24, 1999.

VERMEIRE, E.; HEARNSHAW, H.; VAN ROYEN, P.; DENEKENS, J. Patient adherence to treatment: three decades of research. a comprehensive review. J. Clin. Pharm. Ther., v. 26, p. 331-345, 2001.

WORLD HEALTH ORGANIZATION, 2003. Adherence to long-term therapies: evidence for action. Disponível em: $<$ http://www.who.int/chronic_conditions/en/ adherence_report.pdf $>$. Acesso em: 27 set. 2004.

Recebido para publicação em 22 de maio de 2006 Aceito para publicação em 03 de outubro de 2006 